\title{
Oscillatory regularity of charge carrier traps energy spectra in silicon organic polymer poly(di-n-hexylsilane)
}

\author{
A. Gumenjuk, N. Ostapenko, Yu. Ostapenko, and O. Kerita \\ Institute of Physics National Academy of Sciences of Ukraine, 46 Nauky Ave., Kiev 03028, Ukraine \\ E-mail: nina.ostapenko@gmail.com
}

S. Suto

Departments of Physics, Tohoku University, Sendai 980-8578, Japan

A. Watanabe

Institute of Chemical Reactions, Tohoku University, Sendai 980-8578, Japan

Received April 2, 2012, revised May 12, 2012

\begin{abstract}
Charge carrier traps energy spectra have been investigated in silicon organic polymer poly(di-n-hexylsilane) by fractional thermally stimulated luminescence in the temperature range from 5 to $200 \mathrm{~K}$. The energy spectrum of traps has been found to be discrete in nature, not the quasi-continuous, as it was considered earlier. It has been established that the traps energies form two characteristic series resulting from the vibrational quanta at 373 and $259 \mathrm{~cm}^{-1}$, respectively. It is important that these vibrational quanta coincide with the frequencies of the totally symmetric vibrational modes of silicon chain which are active in Raman spectrum. The regularities mentioned are analyzed using the oscillatory traps model as the basis.
\end{abstract}

PACS: 78.60.Kn Thermoluminescence;

78.66.Qn Polymers; organic compounds.

Keywords: poly(di-n-hexylsilane), charge carrier traps, energy spectrum, fractional thermally-stimulated luminescence, oscillatory model.

\section{Introduction}

In crystals due to the crystal structure periodicity, the energy spectrum of the charge carrier traps is discrete in character. The many organic polymers have amorphous structures, so the traps in them should not create discrete energy states. However, the silicon organic polymers demonstrate certain indications of ordering. Poly(di-nhexylsilane) (PDHS) is a typical representative of polymers of this class. The main polymer chain of PDHS represents a zigzag chain of the $\sigma$-bounded silicon atoms with organic molecules attached to them. The PDHS is known to form polycrystalline films [1] and degree of polymer ordering amounts to $\sim 80 \%$ [2]. The distinct $\mathrm{x}$-ray reflections [3-5], typical for the crystals, as well as the fact that IR [2] and Raman [3,6,7] spectra are composed of narrow bands similar to the crystalline spectra confirm the existence of specific ordering in this polymer. At room and lower temperatures, the PDHS macromolecule has an ordered trans-conformation and can be considered as onedimensional crystal. Due to delocalization of the $\sigma$-bonds
( $\sim 30 \mathrm{Si}$ atoms in length) in the main polymer chain [8], the polymer shows a number of unique photophysical characteristics, namely: strong absorption in the UV region, high quantum fluorescence yield and charge carriers mobility. The features make it possible to use the polymer as transport [9] and light-emitted layers [10] in electroluminescent devices. In real conditions, the all-trans symmetry of polymer chains breaks down because of the rotation of the polymer chains segments around the $\mathrm{Si}-\mathrm{Si}$ bonds and as a result, the formation of conformational defects [1]. The defects create energy barriers for holes; therefore, at low temperatures a hole appears to be localized within a segment, i.e., the segment situated between two neighboring conformational defects. Thus, a special feature of these polymers is that in them the linear defects act as traps whereas in inorganic crystals the point defects play essentially the role of such traps. The presence of translational periodicity in the main polymer chain is supposed to set up prerequisites for the appearance of the discrete energy states of the traps. We carried out an experimental verification of this assumption on the PDHS films using the im- 
proved method of the fractional thermally stimulated luminescence (FTSL) [11]. It has been found that the energy spectrum of the charge carriers traps in PDHS is discrete that is typical for crystals. Distinct from the crystals, the frequency factor demonstrated marked dispersion finally resulting in the broad almost structureless TSL curves (socalled glow curves). In the present paper, the peculiarities of the spectrum are analyzed using the oscillatory model of the charge carrier traps as a basis.

\section{Experimental technique}

The FTSL measurements were carried out with automatic equipment for optical thermally stimulated spectroscopy over a wide temperature range from 5 to $200 \mathrm{~K}$ with the heating rate of $\beta=0.25 \mathrm{~K} / \mathrm{s}$. The PDHS films were fabricated by direct casting from toluene solution on stainless steel substrates. We used several PDHS films with a thickness of $5 \mu \mathrm{m}$ : freshly prepared polymer films, as well as the films irradiated with unfiltered light of $\mathrm{Hg}$ lamp at room temperature before the TSL study. Such irradiation leads to an increase in the traps number due to the polymer chains photodestruction [12]. The charge carriers in the PDHS were photogenerated by the sample excitation by unfiltered light of $\mathrm{Hg}$ lamp for $2 \mathrm{~min}$ at 5 or $60 \mathrm{~K}$.

The FTSL method involves a detection of the emission intensity of the sample pre-excited at low temperature at its linear heating over a small temperature range followed by immediate cooling. Cyclically repeated heating-cooling continues up to total trap emptying. For the initial part of the TSL curve (corresponding to $1-5$ per cent of the existing filled traps) the concentration of the filled traps is small and we can neglect it. The emission intensity can be described by Boltzmann factor as follows

$$
I=I_{0} \exp \left(-E / k_{B} T\right)
$$

where $I_{0}=s n, \quad n$ ( $\approx$ const $)$ is the initial concentration of the filled traps for the current fraction, $s$ is the frequency factor which is traditionally regarded as the carrier frequency of attempts to escape from a trap. The activation energy of traps can be calculated in the terms of Arrhenius coordinates

$$
E=-k_{B} \frac{\Delta(\ln I)}{\Delta\left(\frac{1}{T}\right)} .
$$

The fractional method enables to obtain a set of data for energies of a singly excited sample that significantly increases the reliability of the results. The important advantage of the method is that the calculated energies are independent of the TSL kinetics. Saw-type [13] or step-wise [14] heating modes are traditionally used. Due to an increase in the initial temperature, the initial intensity of each next fraction is higher than that of the previous one; therefore, the maximum numbers of fractions is limited by dynamic range of the reg- istration system (not more than 20-30 fractions in the temperature range of 50-100 K). This substantially reduces the resolution of the fractional method.

We used another regime of the temperature modulation: the sample was cooled down to the temperature at which the glow intensity attained its minimum value (which is subsequently referred as the background emission intensity). The initial slope was registered until the signal intensity reached the value specified by the program. The full dynamical range $\left(I_{\max }=65535\right)$ of the detection system was used to register every fraction. Under such conditions, the number of the fractions obtained is limited only by the detection system sensitivity rather than capacity of analogdigital signal converter. The TSL intensity of the polymer studied is quite high. This makes it possible to increase the sensitivity by $2-3$ order of magnitude as compared with that used for the detection of the integral TSL curves. As a result, one can detect more than 150 fractions in the temperature range of $5-100 \mathrm{~K}$.

An additional feature of the method is a possibility to determine the zero signal level before and after detection of each fraction, i.e., the zero drift for the current fraction was practically eliminated by its linear interpolation. This feature appeared to be important as the fractional curves are detected at high sensitivity of the registration channel and the zero drift occupied a significant part of the dynamic range. Measurement of the dark signal level for each fraction revealed that the TSL was accompanied by a background emission whose level decreased slowly from fraction to fraction. To obtain the correct values of the fraction energies, the background was subtracted when treating the experimental data. More details of the procedure may be found in Ref. 11. The elimination of the background was of crucial importance in determination of the correct values of the fraction energies. For example, the background subtraction, which is only $4 \%$ of the maximum intensity of the fraction, leads to an increase in the energy value by one-third. In this case, the root-mean deviation would be reduced by an order of magnitude.

\section{Results and discussion}

Energy traps spectrum was investigated on freshly prepared PDHS films and the results obtained are shown in Fig. 1 depending on the effective temperature derived from Eq. (3) according to the relation

$$
T_{\text {eff }}=\frac{E}{k_{B} \ln \left(I_{0} / I_{\max }\right)}
$$

where $E$ is energy calculated for the fraction, $I_{\max }$ is the maximum fraction intensity, and $I_{0}$ was found by averaging over all experimental points of the fraction on the basis of Eq. (1). The fraction energies (Fig. 1, curve 2) form plateaus at $0.034,0.046$ and $0.115 \mathrm{eV}$. Measured for the same film integrated TSL curve is shown by a solid line in the 


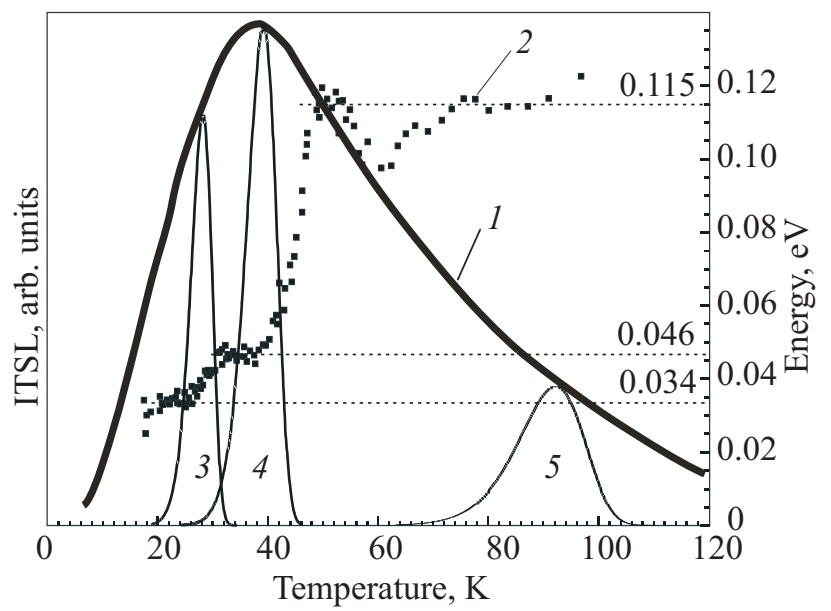

Fig. 1. TSL of freshly prepared PDHS film: TSL curve (1); fractional energies as a function of the effective temperature according to Eq. (3) (2); calculated elementary TSL curves for energies $0.034,0.046$ and $0.116 \mathrm{eV}$ and positions of maxima at the ends of plateau at 28,40 , and $92 \mathrm{~K}$, respectively (3-5).

figure. The curves 3,4 and 5 illustrate the intensities of the TSL elementary curves calculated in terms of linear kinetics for the corresponding plateau energies and positions of the peaks at the plateau ends $(28,40$, and $92 \mathrm{~K})$. These three peaks do not overlap whereas the experimental TSL curve does not show any evidence of the appropriate structure. This fact and the presence of the discrete energy spectra of traps at the same time point to a disperse character of the frequency factor. Thus, the part of the integrated curve obtained over the temperature range, which is specific for each plateau represents a superposition of the elementary TSL curves with the same energy and different frequency factors. Based on these facts, the intensity of the fraction can be regarded as a superposition of the initial parts of the same slope $I=\sum\left(s_{i} n_{i}\right) \times \exp \left(-E / k_{B} T\right)$. It is worthwhile to pay attention at the intensity curve dip at the plateau with the energy of $0.115 \mathrm{eV}$. The reason of this dip appearance is discussed below.

Figure 2 illustrates the results obtained for the PDHS film pre-irradiated by unfiltered light of $\mathrm{Hg}$ lamp at room temperature. In the TSL curve an additional broad band with a maximum at $\sim 125 \mathrm{~K}$ is seen evidencing an appearance of deeper traps in the polymer. The UV light absorption at room temperature is known to result into photodestruction of polymer chains [12], i.e., Si-Si bond breaking. Attachment of the ruptured $\mathrm{Si}$ bond to the $\mathrm{Si}-\mathrm{Si}$ bonds of the neighboring chain gives birth to a new defect, a branch point $[15,16]$. In the film subjected to photodestruction the short segments appear which are consistent with much deeper traps. This conclusion is supported by theoretical calculations made in [17]. Comparison of Fig. 1 and Fig. 2 shows that formation of additional conformational defects does not cause the broadening of the energy trap spectra but only produces new deeper traps.

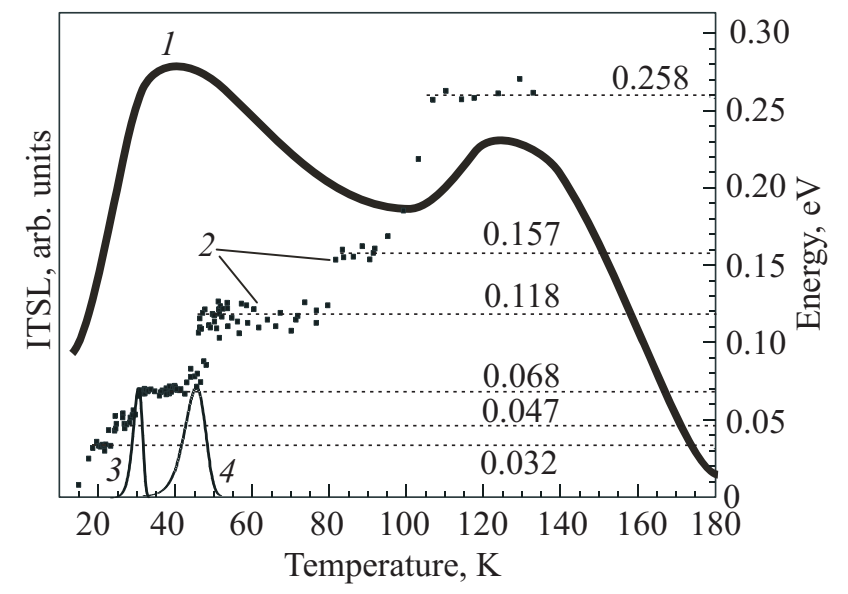

Fig. 2. TSL for PDHS film pre-irradiated with UV light; TSL curve (1), temperature dependence of the energy spectrum of charge carrier traps (2); calculated TSL elementary curves for the energy $E=0.068$ and positions of maxima at the beginning and at the end of the plateau at $T_{\max }=31$ and $47 \mathrm{~K}$, respectively $(3,4)$.

Besides the traps with the energies of $0.032,0.047$ and $0.0116 \mathrm{eV}$ found in freshly prepared sample new traps with the energies of $0.068,0.157$ and $0.258 \mathrm{eV}$ are observed too. Curves 3, 4 in Fig. 2 were calculated for linear kinetics in such a way that their peaks would lie at the beginning and at the end of the plateau with the energy of $0.068 \mathrm{eV}$. The frequency factors calculated from the expression

$$
s=\frac{E \beta}{k_{B} T_{m}^{2}} \exp \left(\frac{E}{k_{B} T_{m}}\right)
$$

$\left(E=0.068 \mathrm{eV}, \beta=0.25 \mathrm{~K} / \mathrm{s}, T_{m}-\right.$ the peak temperature), are $1.9 \cdot 10^{10} \mathrm{~s}^{-1}$, and $1.4 \cdot 10^{6} \mathrm{~s}^{-1}$, respectively. The difference between these values, being as much as four orders of magnitude, points to a substantial dispersion of the frequency factor in the polymer studied.

We assumed that the dip in the energy spectrum of traps over the range of 55-75 K (Fig. 1) is due to simultaneous emptying of two traps with different energies. To prove the suggestion the study of the FTSL has been carried out with freshly prepared polymer film excited at $60 \mathrm{~K}$ to "clean" low-temperature peaks. This temperature corresponds to the beginning of the dip on curve 2 in Fig. 1. In so doing shallow traps with activation energies of 0.034 and 0.046 $\mathrm{eV}$ remained empty as well as some traps with the energy of $0.115 \mathrm{eV}$ and high value of the frequency factor. In Fig. 3 fractional energies are shown that were obtained in the experiment. It is clearly seen a new plateau at $0.081 \mathrm{eV}$ along with plateau at $0.116 \mathrm{eV}$ present in two previous Figures. Thus, the presence of the dip in the energy spectrum of traps over the range of 55-75 K (Fig. 1) is associated with simultaneous emptying of the traps with the energy $0.081 \mathrm{eV}$ and some traps of the energy $0.115 \mathrm{eV}$, which are characterized by high frequency factor. 


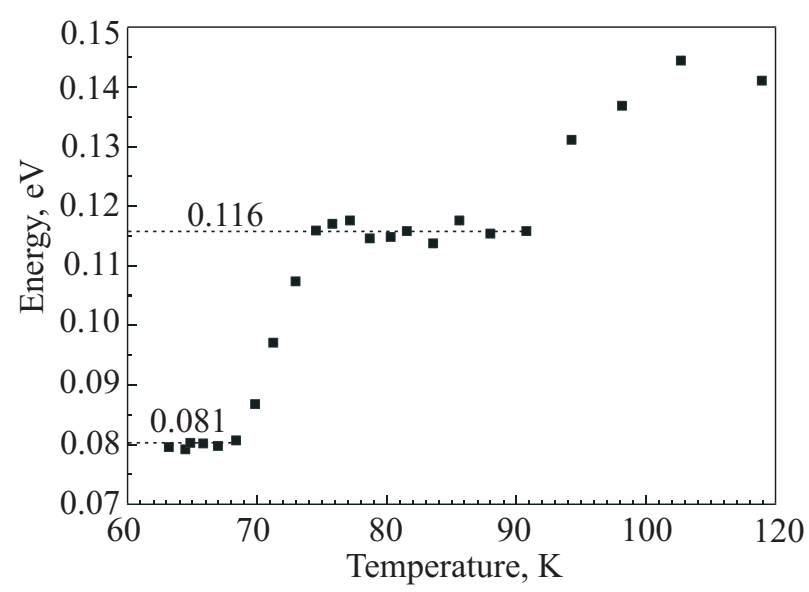

Fig. 3. Temperature dependence of the energy spectrum of charge carrier traps of the freshly prepared PDHS film excited at $T=60 \mathrm{~K}$.

Analysis of the experimental results obtained showed that the activation energies of the charge carrier traps in PDHS can be fitted by the two oscillatory series given in Table 1. The corresponding experimental values of energies are listed in the second and fourth columns of the table. The first column shows the ratios between the experimental energy values (column 2) and value of $E=0.047 \mathrm{eV}$ rounded off to the half-integer values $\left(n^{\prime}\right)$. The resulting energy of vibrational quantum was determined by averaging $E_{\exp } / n^{\prime}$ values providing the magnitude of $\hbar \omega_{1}=$ $=0.0462 \pm 0.0004 \mathrm{eV}\left(373 \mathrm{~cm}^{-1}\right)$ for the first series. In the third column, the values are given for the energies calculated from the generalized formula for harmonic oscillator

Table 1. Oscillatory series of trap energies in PDHS films

\begin{tabular}{c|c|c|c|c}
\hline \hline \multirow{2}{*}{$\begin{array}{c}\text { Multiplicity } \\
n^{\prime}=E / \hbar \omega\end{array}$} & \multicolumn{2}{|c|}{$\begin{array}{c}\omega_{1}=0.0462 \\
373 \mathrm{~cm}^{-1}\end{array}$} & \multicolumn{2}{c}{$\hbar \omega_{2}=0.033$} \\
& $E_{\exp }$ & $E_{\text {clc }}$ & $E_{\exp }$ & $E_{\text {clc }}$ \\
\cline { 2 - 5 } & 0.047 & 0.0462 & 0.033 & 0.033 \\
1 & 0.068 & 0.069 & - & 0.049 \\
1.5 & 0.116 & 0.112 & 0.081 & 0.082 \\
2.5 & 0.159 & 0.163 & 0.116 & 0.115 \\
3.5 & - & 0.208 & - & 0.145 \\
4.5 & 0.257 & 0.254 & - & 0.181 \\
5.5 & \multicolumn{3}{|c}{} \\
\hline \hline
\end{tabular}

$$
E=\hbar \omega n^{\prime}
$$

where $n^{\prime}$ can take both integer and half-integer values. There is a good agreement between the experimental and calculated values. The second oscillatory series generated by the vibrational quantum of the energy $\hbar \omega_{2}=0.0328 \mathrm{eV}$ is represented by three experimental values in the fourth column. In the last column, the energies are given calculated from Eq. (5). When comparing the data from the columns 3 and 5 , it is seen a superposition of some energies of the two series. Just the energy of $0.082 \mathrm{eV}$ does not have an equivalent in the first series. It is noteworthy a good agreement between the first terms of both series and corresponding vibrational quanta whereas the rest ones appear to be multiples of half-integer values of the quanta. Once again, there is a good correlation between the experimental and calculated values.

The validity of the energy values obtained as well as the existence of oscillatory series for these energies are supported by a coincidence of the energies, which the both series are based on, with the lines in Raman spectrum of PDHS at 259 and $373 \mathrm{~cm}^{-1}(0.0321$ and $0.0462 \mathrm{eV}$, respectively), which stem from the totally symmetric vibrational modes of the silicon chain $[3,6,7]$.

When constructing a model of transitions which are used to explain the presence of the oscillatory series of traps energies in PDHS polymer, we will rely upon the following well-known positions.

1. Silicon chain segments of different length are the traps for holes, the maximum segment length is equal to 5 $\mathrm{nm}$ [8]. The boundaries between the segments are the breaking of translational symmetry of silicon chain due to rotations of the segments around the $\mathrm{Si}-\mathrm{Si}$ bonds at different angles as well as defects of branching type $[1,15,16]$. The holes created by UV radiation at $5 \mathrm{~K}$ are situated on the segments whose boundaries are the potential barriers for their transport along the polymer chain.

\section{Trapped holes form polarons.}

The oscillatory series in the energy spectrum of traps was earlier observed in inorganic crystals. For example, in sapphire [18] and in trichloride of cesium-cadmium [19] one series and two series were found respectively, with different values of $\hbar \omega$. In $\mathrm{Zn}$ tangstate [20] and $\mathrm{Cd}$ tangstate [21] were observed three series in each. In yttrium aluminum garnet [22] five oscillatory series were found. In this case the vibrational energies of the quanta coincide with the energies of lines corresponding to the vibrational modes, which are active in Raman spectra. Based on these experiments it was concluded that in these crystals the energies of traps are always multiples of the half-integer number of vibrational quanta $\hbar \omega$ whereas in alkali halide crystals [23-25] the energies of the most part of traps appeared to be multiples of integer value of the vibrational quanta. The occurrence of one or other type of regularity, i.e., whether or not the trap energies are multiples of integer or half-interger value of the vibrational quantum energy $\hbar \omega$ depends on the peculiarities of polaron-lattice interaction. The normalized probability of the vibrational state polaron with $E=\hbar \omega(N+1 / 2)$ population can be calculated from Eq. (6).

$$
w_{N}=g_{N} \frac{\exp \left[-\frac{\hbar \omega(N+1 / 2)}{k_{B} T}\right]}{A}
$$

where $N$ is the vibrational quantum number, which allows the polaron to be released. 
Here $A$ is the statistical sum defined by normalization of the probability to $1, g_{N}$ represents statistical weight. If the pure oscillatory mechanism of the released charge carrier transport to a recombination center is realized, then, taking into account that $g_{N}=1$ for one-dimensional case, we can calculate

$$
A=\exp \left(-\hbar \omega / 2 k_{B} T\right) \frac{1-\exp [-(N+1) \hbar \omega / k T]}{1-\exp (-\hbar \omega / k T)} .
$$

For $\hbar \omega=0.046 \mathrm{eV}$ and $T=50 \mathrm{~K}$ we obtained: $\exp (-\hbar \omega / k T)=2 \cdot 10^{-5}<1$, hence

$$
w \sim \exp \left(-\frac{N \hbar \omega}{k_{B} T}\right)
$$

i.e., the trap energy appears to be multiple of an integer number of the vibrational quanta. If the collective state is added to the oscillatory one, then they both contribute into statistical sum, i.e. $A=A_{\mathrm{osc}}+A_{\mathrm{del}}$. As the evaluation [24] indicates, the collective component exceeds the oscillatory one by several orders of magnitude due to its statistical weight being much greater. Hence, the oscillatory component may be neglected and energy of the trap can be described by a half-integer value $\hbar \omega$, as

$$
w \sim \exp \left[-\frac{(N+1 / 2) \hbar \omega}{k_{B} T}\right] .
$$

The half-integer value of the trap energy suggests that collective state is involved in the transport of the charge carriers to the recombination center. The integer energy value means direct tunneling of the charge carrier to the excited state of a nearby recombination center.

The abovementioned arguments are applicable for analyzing the peculiarities observed in the energy spectrum of traps in the PDHS polymer. Then a pure oscillatory mechanism is realized for two shallow traps with the energies of $\hbar \omega_{1}=0.046 \mathrm{eV}, \hbar \omega_{2}=0.033 \mathrm{eV}$ whereas for much deeper traps account must be taken of the occurrence of collective mechanism of their transport to recombination centers. Further analysis requires an assumption to be made as to localization point of the recombination partner - electron. The conformational defect creates barrier for a hole but it is precisely the potential well for an electron. In the excited polymer the holes are delocalized within the segment in HOMO while the electrons are localized on the conformational defects in LUMO. If the electron is localized at the end of the segment with a hole, then after a transition into excited vibrational state $(n=1)$ the hole may tunnel through the barrier (which is narrower than that for the $n=0$ state) and recombine with the electron. In this case, the activation energy is equal to a quantum of the phonon energy. It is important that if an electron is present at the segment boundary the barrier height for the hole is considerably lower due to the electron Coulomb field, therefore, for the recombination of the hole with the electron it is sufficient for the hole to be excited to the first vibrational level. This fact explains the absence of energies with the multiplicity values of $\hbar \omega$ more than 1 . In the case of an electron absence at the segment ends wherein the hole is, then the hole should get enough energy to go to a neighboring segment. If the electrons situated at the ends of the segment, the recombination occurs, i.e., the process described above is repeated.

Considering the thermal release of hole as a transition of oscillator to high vibrational levels, it should be remembered that in this case the polarization surroundings of the hole serves the function of oscillator. An important point is that the symmetry of polarization distortion and the vibrational mode interacting with the hole should be the same. As a consequence, on absorption of the sufficient phonon quantities the situation may occur for some phase of the vibrational process when the position of ions vibrating may come close to their equilibrium position in the absence of the hole as a reason of polarization. The hole overcoming the barrier gains kinetic energy very fast and since the inertia of the ion subsystem is considerably larger than that of hole, the hole accelerates at essentially invariable ions positions (adiabatic approximation). The fast hole does not have an opportunity to polarize the ion subsystem and by the time of scattering it can be considered as Bloch particle. Based on the analysis given above this process can be described by the activation energy with half-integer value of the vibrational quantum.

Hence, in the polymer under study the "classical" trap depth, i.e., the distance between the top and the bottom of the potential well should vary randomly because of a variation in the segment length and properties of conformational defects (turns on the different angles and presence of branching points). However due to the polaron effect the potential well is filled with the vibrational levels from which the holes are released. As a result, the activation energy of the hole appears to be smaller than the full depth of the potential well.

The rate of hole release from a particular vibrational level (i.e., FTSL intensity) depends on the degree of level population (the Boltzmann factor), and the transparency of the barrier which is included in the frequency factor. The holes tunneling occurs basically from all excited vibrational levels, but the dominant ones are transitions from the highest vibrational level of polaron which is matched by the most transparent barrier. Besides, the transitions from the ground state are quite probable though for this state the transparency of the barrier is quite low but the state population is the greatest. In the context of the model proposed the background emission may be treated as tunnel transitions from the ground vibrational state of polaron $n=0$. 


\section{Summary}

The study of FTSL from polymer PDHS films revealed a discrete character of the traps energy spectrum. Moreover the energies of the traps form two oscillatory series with the energies of vibrational quanta, underlying the series, being consistent with the energies of total symmetric vibrational modes of silicon chain of the polymer which are active in Raman spectrum. In this regards PDHS resembles inorganic crystals with the ionic-covalent type of bonding for which the occurrence of the oscillatory dependence of the traps energy spectrum has been properly established. The presence of long segments of PDHS polymer chains with trans symmetry gives reason to suggest the existence of lattice phonons. At the same time, the interaction of holes with a regular ionic environment leads to discrete energy state of traps of vibrational nature, that is the equidistant energy levels of the hole polaron. Phonons arising due to increase in temperature are absorbed by the hole polaron, resulting into transition to the higher excited states. It is worthwhile to notice that phonon energy $\hbar \omega$ is precisely consistent with the energy difference between two neighboring levels of the state of the polaron. Since the holes release occurs from the vibrational levels of the system, it determines the discrete spectrum of the traps whose energies correlate with the energy of vibrational modes of silicon chain.

1. M. Pope and C.E. Swenberg, Electronic Processes in Organic Crystals and Polymers, Oxford University Press, N.Y. (1999), p. 877.

2. M.M. Despotopoulou, R.D. Miller, J.F. Rabolt, and C.W. Frank. J. Polym. Sci. B34, 2335 (1996).

3. J.F. Rabolt, D. Hofer, R.D. Miller, and G.N. Fickes, Macromolecules 19, 611 (1986).

4. H. Kuzmany, J.F. Rabolt, B.L. Farmer, and R.D. Miller, J. Chem. Phys. 85, 99164 (1986).

5. C.A. van Walree, T.J. Cleij, L.W. Jenneskens, E.J. Vlietstra, G.P. van der Laan, M.P. de Haas, and E.T.G. Lutz, Macromolecules 29, 7362 (1996).
6. S.S. Bukalov, L.A. Leites, G.I. Magdanurov, and R. West, J. Organomet. Chem. 685, 51 (2003).

7. L.A. Leites, S.S. Bukalov, T.S. Yadritzeva, M.K. Mokhov, B.A. Antipova, T.M. Frunze, and V.V. Dement'ev, Macromolecules 25, 2991 (1992).

8. Y.R. Kim, M. Lee, J.R.G. Thorne, R.M. Hochstrasser, and J.M. Zeigler, Chem. Phys. Lett. 145, 75 (1988).

9. H. Suzuki, H. Meyer, S. Hoshino, and D. Haarer, J. Appl. Phys. 78, 2684 (1995).

10. A. Sharma, M. Katiyar, Deepak, S. Seki, and S. Tagawa, Appl. Phys. Lett. 8, 143511 (2006).

11. A. Gumenyuk, N. Ostapenko,Yu. Ostapenko, O. Kerita, and S. Suto, Chem. Phys. 394, 36 (2012).

12. Yu.A. Skryshevskiy and A.Yu. Vakhnin, Phys. Status Solidi 43, 569 (2001).

13. H. Gorbecht and D. Hofmann, J. Phys. Chem. Solids 27, 509 (1996).

14. I.A. Tale, Phys. Status Solidi A66, 65 (1981).

15. Y. Kanemitsu and K. Suzuki, Phys. Rev. B51, 13103 (1995).

16. A. Watanabe, M. Nanyo, T. Sunaga, and A. Sekiguchi, $J$. Phys. Chem. 105, 6436 (2001).

17. A.R. Porter, M.D. Towler, and R.J. Nedds, Phys. Rev. B64, 035320 (2001).

18. A.F Gumenjuk., S.Yu Kutovyi, and M.O Grebenovich, Funct. Mater. 9, 314 (2002).

19. G.P. Blinnikov, V.N. Golonchka, and A.F. Gumenjuk, Opt. Spectroscop. 69, 1054 (1990).

20. I.S. Gorbań, A.F. Gumenjuk, and S.Yu. Kutovyi, Ukr. J. Phys. 40, 47 (1995).

21. A.F. Gumenjuk, S.Yu. Kutovyi, and O.B. Okhrimenko, $U k r$. J. Phys. 42, 870 (1997).

22. I.S. Gorban, A.F. Gumenjuk, W.Ya. Degoda, and S.Yu Kutovyi, Opt. Spectroscop. 75, 47 (1993).

23. A.F. Gumenjuk and S.Yu. Kutovyi, Centr. Europ. J. Phys. 1, 307 (2003).

24. A.F. Gumenjuk and S.Yu. Kutovyi, Ukr. J. Phys. 50, 1125 (2005).

25. A.F. Gumenjuk, S.Yu. Kutovyi, W.G. Pashchenko, and O.P. Stanowyi, Ukr. J. Phys. 54, 999 (2009). 